\title{
The Nature and Units of Social Selection
}

\author{
Geoffrey M. Hodgson and Thorbjørn Knudsen
}

16 March 2006

Published in the Journal of Evolutionary Economics

The Business School, University of Hertfordshire, De Havilland Campus, Hatfield, Hertfordshire AL10 9AB, UK and

Department of Marketing and Management, University of Southern Denmark, Odense Campus, 5230 Odense M, Denmark

Address for correspondence:

Malting House, 1 Burton End, West Wickham, Cambridgeshire CB1 6SD, UK

g.m.hodgson@herts.ac.uk

KEY WORDS: Subset selection, generative selection, generalized selection, Price equation

JEL classification: B25, B52, D20, D83, L20

\begin{abstract}
On the basis of the technical definition of selection developed by George Price (1995), we describe two forms of selection that commonly occur at the social level, subset selection and generative selection. Both forms of selection are abstract and general, and therefore also incomplete; both leave aside the question of explaining the selection criterion and why entities possess stable traits. However, an important difference between the two kinds of selection is that generative selection can accommodate an explanation of how new variation is created, while subset selection cannot. An evolutionary process involving repeated cycles of generative selection can, in principle, continue indefinitely because imperfect replication generates new variation along the way, whereas subset selection reduces variation and eventually grinds to a halt. Even if the two kinds of selection are very different, they share a number of features. First, neither subset selection nor generative selection implies improvement: neither kind of selection necessarily leads to efficiency or implies systematic outcomes. Second, both subset selection and generative selection can lead to extremely rapid effects in a social population. Third, in the social domain, both generative selection and subset selection involve choice and preference in some way: neither form of selection necessarily excludes intentionality. In concluding the article, we single out a challenge for future research in identifying the role of various units of culture in selection processes and the multiple levels at which social selection processes take place.
\end{abstract}




\title{
The Nature and Units of Social Selection
}

\author{
Geoffrey M. Hodgson and Thorbjørn Knudsen
}

\section{Introduction}

In common usage, selection is synonymous with choice, connoting the act of selecting, as in the selection of an item from a restaurant menu. ${ }^{1}$ In contrast, the scientific usage of selection has a very precise meaning, referring to a change in the distribution of a population property (a set of traits) such as the colour of moths or the size of firms in an industry. However, as emphasized below, this scientific meaning does not necessarily exclude choice.

Two very different concepts of selection are employed in science, and both are encompassed by its general mathematical definition (Price, 1995; Knudsen, 2002, 2004). One meaning can involve the selection of a subset of elements from a set. Examples include the selection of a subset of reindeer that survive a predator's attack or the selection of a subset of firms that survive an industry shakeout. Price (1995) termed this subset selection. Subset selection is very different from the concept of generative selection - which was part of Darwin's great achievement - where offspring are not subsets of parents. ${ }^{2}$

The general concept of selection follows from a mathematical definition developed by George Price (1995). It can be described in the following way: Selection involves an anterior set of entities, each interacting with their environment, and somehow being transformed into a posterior set, where all members of the posterior set are sufficiently similar to some members of the anterior set, and where the resulting frequencies of posterior entities depend upon their properties in the environmental context. ${ }^{3}$

The concept of selection that follows from Price's technical definition is very general and includes a number of interesting and complicated cases. We shall focus on particularly important

1 The authors are very grateful to Guido Bünstorf, Werner Callebaut, Christian Cordes, Peter Corning, J. Stanley Metcalfe, Janet Landa, Joel Mokyr, Richard R. Nelson, Peter Richerson, Jack Vromen, Ulrich Witt, and other participants of the workshop on "Evolutionary Concepts in Economics and Biology" arranged by the Evolutionary Economics Group, at the Max Planck Institute, Jena, 2-4 December 2004.

2 Nevertheless, we chose the term 'generative selection', rather than 'Darwinian selection', because both forms of selection are present in Darwin's work.

3 The technical definition of selection is explored at greater length elsewhere (Andersen, 2004; Frank, 1998; Hofbauer and Sigmund, 1998; Pepper and Knudsen, 2001; Price, 1995; Knudsen, 2002, 2004; Henrich, 2004) and summarized in the Appendix below. 
objects of selection, such as interactors (e.g. organisms or firms) or replicators (e.g. genes or routines). For expository purposes we define each anterior set to include and exhaust one class of entities, such as a species (a class of organisms) or a population of firms (a class of institutions). This implies that we only consider the case where members of the anterior set are eliminated through extinction. New entities can appear in the posterior set, but only in consequence of a replication process. Particular entities do not reappear after they have gone extinct.

Through selection, a set of entities, a population, will gradually adapt in response to a complex of environmental factors. Both conceptually and empirically, the change caused by selection is sharply distinguished from such change that happens when information is transferred, in processes where an entity develops, or as a result of individual experimentation. Such transmission and innovation effects alter the property of individual entities when the population is held constant. In contrast, selection alters the composition of the population, but holds constant the properties of the individual entities.

There are number of advantages of the general definition of selection presented here. First, it settles the meaning of selection so that much idle speculation can be avoided. Second, it is stated in an abstract and general way that makes no reference to any particular empirical domain, be it physical, biological or social. Third, it encompasses both subset selection and generative selection.

It should be emphasized, however, that while this general definition of selection involves a systematic relationship between the anterior set and the posterior set, the selection outcome is not necessarily an improvement, or close to the global optimum. Consequently, this general conception of the selection process is not necessarily systematic in the sense of leading to efficacious outcomes. Although successive adaptations to an environment can often be observed at the social level as well as in nature, there are cases such a lock-in, path dependence and frequency dependence that confine the selection process to a suboptimal track.

\section{Subset Selection}

Consider the following example of subset selection in nature (Landa et al. 1999). There is an anterior distribution of speed in a population of reindeer. A wolverine slays a number of the reindeer. In consequence, the distribution of speed in reindeer is transformed into a posterior set. If the slowest die, there is a significant and positive selection for higher average speed in reindeer. However, subset selection could as well occur because an avalanche eliminated reindeer at random, or perhaps even the fastest reindeer. A similar argument holds for subset selection on firms and other institutions. Subset selection can alter the value of a population property when some properties of firms remain stable (such as the number of hierarchical layers) in a population of firms. For example, business ventures that are eliminated because of bankruptcy are a case of subset selection. This is a very common phenomenon. At any given time, only a fraction of new entrepreneurial start-ups survive (Aldrich, 2004; Katz and Gartner, 1988).

Subset selection is here defined as: one cycle of environmental interaction and elimination of entities in a population, so structured that the environmental interaction causes elimination to be differential. Evolution happens because of repeated cycles of subset selection. Each cycle of subset selection eliminates variation. In a formal description, subset selection is a contraction mapping. Each cycle of subset selection contracts the anterior set, yielding a posterior set with fewer elements than the anterior set. Eventually, after many cycles of subset selection, the anterior set has 
contracted so it only contains one remaining member of the population - or it may even become an empty set. Subset selection will eventually run dry of variation and come to a halt.

Selection is always a causal claim. In the case of subset selection, the causes of differential survival must be traced to environmental interaction. For example, there is a critical difference between reindeer being killed by wolverines and by avalanches. In both cases, the causes of differential survival are properly identified, but the outcome is likely to be very different. Mere observations of differential survival are referred to as sorting (Vrba and Gould, 1986). They can obviously be quite misleading. Unless qualified by identification of the operative selection criteria, there is no way of knowing whether observed survival rates reflect selection processes, migration or something else. Notably, selection criteria can operate on different time-scales and thus appear more or less systematic - wolverines kill reindeer more frequently than do avalanches and thus appear more systematic.

Surprisingly many population processes in nature and at the social level involve subset selection. Molecules, cells, plants, moths, reindeer, and tigers are populations of biological interactors whose properties are altered by subset selection. Hard winters, hot summers, and various natural catastrophes commonly eliminate organisms. Similarly, institutions, social organizations and human individuals are populations of social interactors whose properties are altered by subset selection. As previously noted, the elimination of business organisations through bankruptcy is a very common example of subset selection at the social level. Through elimination of entities, subset selection also shapes the properties of populations of biological replicators, such as genes, and social replicators, such as habits and routines.

In cases where the selection criterion is located at the physical or biological level, such as earthquakes, subset selection does not involve choice and preference. However, when the selection criterion is located at the social level, human choice and preference is always somehow involved, even if indirectly. A common instance of subset selection at the social level is the elimination of firms through bankruptcy. Weak technical efficiency, wanting market orientation, a reputation for dishonesty, a lack of institutional support caused by inadequate business models, inefficient production processes, unattractive product portfolios, and ineffective internal organization often lead to bankruptcy (Aldrich, 1999; McMillan, 2002; Tushman and Anderson, 1986). In such cases, choices and preferences are involved in some way because it is the deliberate choices of managers that lead to failure. However, the selection outcome overall may not itself be a direct reflection of the discrete decision of a single person or group. ${ }^{4}$

Often, the selection criterion operating on firms comprises a complex of environmental factors; it becomes a composite weighted measure. Further complications are introduced by considering the environment as endogenous to the selection process. Powerful (groups of) entities can sometimes change the selection rules. The matter is further complicated because selection of firms is a process operating at multiple interdependent levels. Even though such complications introduce both multiple, recursive and nested components in the selection criterion (fitness function) - thus complicating the unique identification of the causes of a change in a population property - they can readily be accommodated in Price's formulation (see Appendix).

\footnotetext{
4 When a selection outcome overall does reflect such a discrete decision, this is sometimes described as 'artificial selection'. However, contrary to Commons (1934) and many others, artificial selection is not opposed to Darwinian selection (Hodgson, 2002). On the contrary, Darwin (1859) used the concept of artificial selection to illustrate natural selection. Commons was also mistaken in his suggestion that if selection is not artificial then it necessarily excludes choice and will. His critique of Veblen in this respect was thus misguided.
} 
While Price's formulation can accommodate a realistic description of subset selection at the level of firms, there is a further requirement that firms must possess stable traits. Any kind of selection process operates on stable population properties. According to Winter (1971), Nelson and Winter (1982), Hannan and Freeman (1989) and many others, routinized rules are such stable components.

Environmental feedback effectively selects among firms with stable properties; some survive while others are eliminated through bankruptcy. In consequence of market feedback, firms go bankrupt and exit the population (McMillan, 2002). As firms exit the population, the average value of a population property held by these firms can change. Should firms with many hierarchical layers exit, there is a decrease in the average number of hierarchical layers in the population. Such change in a population property because of differential elimination of firms is a common example of subset selection at the social level. The argument extends to the selection of industries, nation states and even entire civilizations.

There are many cases of subset selection where the members of the anterior set are not eliminated through extinction. These cases raise additional conceptual problems that are not considered in the present article. Neither do we consider subset selection in populations of geological entities or artefacts such as stones, pottery, furniture, and books. While these examples of subset selection are encompassed by Price's definition of selection, they do not involve interactors or replicators. The consideration of selection processes where members of the anterior set are not eliminated through extinction or selection processes on entities that are not replicators or interactors is left for future work.

Subset selection offers an explanation of change, but it is an incomplete explanation. In one of the above examples, a wolverine killed the slowest reindeer, but it could as well have slain the fastest. ${ }^{5}$ Subset selection leaves unexplained the selection criterion that transforms the anterior set into a posterior set. The change in the population property (e.g. speed in reindeer) is left unexplained.

The above examples highlight a number of important points. First, many population processes in nature and at the social level involve subset selection where a population property is changed by a selection criterion removing members of a population. Second, subset selection leaves aside the question of explaining the selection criterion and the mechanisms of selection. The elimination and extinction of reindeer or firms must be accounted for by additional specific mechanisms. Third, subset selection does not imply improvement. As noted above, subset selection is formally a contraction mapping. Additional requirements are needed to ensure that the contraction of an initial anterior set containing entities with a distribution of a population property eliminates all but the entity with the globally maximum value of the population property (e.g. the fastest reindeer). Selection would occur even if the wolverine killed the swiftest reindeer or a dictator eliminated the most efficient firms. Fourth, subset selection can lead to extremely rapid effects in a social population. A distribution of firms could be changed within days.

The example also assumed some variation in reindeer speed even though each cycle of subset selection removes variation. Subset selection decouples the generation of new variation from the process of selection and leaves unexplained the variation in the anterior set. In the absence of any additional processes to replenish lost variation, subset selection will eventually come to a halt or can

5 Landa et al. (1999) report from empirical observation that wolverines actually tend to kill less agile reindeer and sheep. 
even lead to the extinction of a population. The example further assumed that the population property in question was stable, that reindeer could not alter their (maximum) speed.

Similar additional assumptions regarding the selection criterion, the generation of new variation and a stable population property justified the example of subset selection on a population of firms. Subset selection assumes a selection criterion that alters the distribution of a population property, it assumes variation in this property, and it assumes that the property in question is stable. Subset selection offers no place for particular explanations of these assumptions. It is an incomplete explanation.

The explanatory incompleteness of subset selection can be viewed as a price paid for its generality. Subset selection can be invoked as an explanation of change in all kinds of distributions that are shaped by a selection criterion, including the selection of interactors (e.g. biological organisms and social institutions) and replicators (e.g. habits, routines, and folkways), the kind of entities which are the primary focus of the present article.

\section{Generative Selection}

In generative selection, the change in a population property, such as moth colour, happens because the interaction of moths with their environment gives rise to differential replication. Moths with better camouflage are less likely to be eaten by predators and therefore, on average, leave more offspring carrying their genes. In this way, dark moths become more frequent in polluted areas. When smokeless zones are introduced so trees' hues become lighter, the colour of the moth population can again become lighter in response.

Generative selection is here defined as: one cycle of replication, variation and environmental interaction so structured that the replication process causes new variation (i.e. new, emergent varieties alter the distribution of the population property) and the environmental interaction causes replication to be differential. ${ }^{6}$ Evolution happens because of repeated cycles of generative selection. Notably, the generation of new variation is integral to generative selection. New variation is generated because replication is never perfect and because replication involves recombination of existing variants.

Consider generative selection on reindeer. There is an anterior distribution of speed in reindeer. Environmental interaction transforms the anterior distribution of speed into an intermediate set (some reindeer die, some are less attractive mating partners), which is then transformed into a new posterior set by replication. There is replacement of entities because of death and replication. Replication involves genetic inheritance between parent and offspring. ${ }^{7}$ More speedy reindeer on average have more speedy offspring. The faster mating partners have a higher survival rate and therefore replicate more, resulting in a significant increase of fast reindeer in the posterior set.

\footnotetext{
6 This definition of generative selection is based on Hull et al. (2001). Generative selection is a particularly important member of the set of distinctive selection processes encompassed by Price's (1995) definition, but he does not use the term.

7 The definitions of selection, replication and interaction employed here do not in principle exclude the 'Lamarckian' possibility of the inheritance of acquired characters. Indeed, Darwin himself believed such 'Lamarckian' inheritance existed. We critically appraise the applicability of the 'Lamarckian' label to socio-economic evolution elsewhere (Hodgson and Knudsen, forthcoming).
} 
There is positive selection of reindeer with high speed and a complementary selection for genes with high speed. ${ }^{8}$ However, generative selection could as well occur because a complex of factors influenced the environmental interaction of reindeer. Attention to unusual environmental factors and care for offspring could influence replication to a larger extent than the ability to run at fast pace.

As in the case of subset selection, generative selection leaves aside the question of explaining the selection criterion and the existence of a stable population property. The nature of the feedback from environmental interaction to replication must be accounted for by additional specific mechanisms, and so must the stability of the population property that we are studying (unless the stability comes from genetic endowment).

However, in contrast to subset selection, generative selection can accommodate an explanation of how new variation is created. Because of replication error (mutation) and genetic recombination, the offspring of reindeer can have new properties that differ from those possessed by their parents. More generally, whenever replication processes produce imperfect copies, generative selection generates new variation. ${ }^{9}$

Generative selection applies to replicating populations in which continuity is secured by the transfer of information (e.g. the transfer of units of culture or genetic inheritance), whereas subset selection applies to populations in which continuity is secured by enduring identity among a subset of entities. In generative selection, each instance of replication creates new variation because of imperfect replication and recombination. In subset selection, additional assumptions and explanations must be invoked in order to explain why new variation is generated as required to continue the evolutionary process. A third stage of processes is needed to replenish lost variation (Foster and Metcalfe 2001). For this reason, systems in which selection processes have remarkable endurance are likely to involve replication (Maynard Smith and Szathmáry 1999).

\subsection{Generative Selection at Multiple Levels in the Social Domain?}

Generative selection is a general principle relating interaction to replication such that new variation is generated in the process of replication. An evolutionary process involving repeated cycles of generative selection can, in principle, continue indefinitely because imperfect replication generates new variation along the way, whereas subset selection removes variation and eventually grinds to a halt. Because of the remarkable endurance of some forms of culture, it is unlikely that they have been sustained by subset selection. We therefore expect that generative selection sustains some evolutionary processes in the social domain.

Possibly, generative selection is present at multiple interdependent levels in the social domain. In order to address this important issue, a definition of the two basic components of generative selection, replication and interaction must be provided. Identification of a hierarchy of social

\footnotetext{
8 The distinction between selection of and selection for is developed by Sober (1984) and others. Note that it involves a distinction between phenotype (of) and genotype (for), but this distinction is not itself expressed within the Price definition of selection.

9 There are two broad additional sources of new variation, drift and environmental changes. Drift is differential replication without environmental interaction. When drift occurs, there is generation of new variation that can fuel subsequent selection processes. Environmental changes can also introduce new variation in a number of ways. For example, environmental changes can alter replicators (e.g. radioactive radiation causing mutations) or influence replication processes so new variation is introduced (e.g. by altering ecological niches).
} 
organization at which selection processes occur requires that we single out a social interactor at each level (Brandon, 1998, 1999; Hull, 1988, 2001). That is, we must provide a definition of a social interactor and its corresponding replicator if we wish to succeed in identifying a possible hierarchy of generative selection processes in the social domain (or rejecting such a claim). In order to get a firmer conceptual grip on the identification of generative selection processes, we must understand the nature of its two basic components, replication and interaction.

The present section therefore begins by defining replication and interaction. It then continues to briefly reiterate what we presently know about units of culture that are social replicators and interactors. Indeed, very little is presently well-defined about the empirical status of social replicators and interactors, and even less is established about the multiple levels at which social selection processes might occur. In consequence, we articulate a challenge for future research in identifying a hierarchy of multiple levels of selection in the social domain.

\subsubsection{Replication and Interaction}

Replication is a process involving a source and a copy with the following features (Sterelny et al. 1996; Godfrey-Smith, 2000; Sperber, 2000; Aunger, 2002): (1) causation: the source must be causally involved in the production of the copy, at least in the sense that without the source the particular copy would not be created, (2) similarity: the copy must possess the capacity to replicate and be like its source in other relevant respects, and (3) inheritance: the process that generates the copy must obtain the information that makes the copy similar to its source from that same source.

Note that replication is generally imperfect - there is similarity but not identity between source and copy,- and it can therefore generate new variation. Also note that, according to criterion (2), the word "copy" is used merely to describe similarity between members of the anterior and posterior set in a selection process. One should not mistakenly infer that a literal copying process is necessarily involved where new entities are (imperfectly) read off of a template or mould. According to criterion (1), the source is causally involved in producing a copy, but the source does not necessarily function as a template. The source could have a minimal role in triggering a construction process leading to a similar entity. For example, it is possible to replicate the habit of using chopsticks in a number of ways, e.g. instruction by a chopstick teacher, imitation of a chopstick user, or reconstruction after seeing a movie featuring a chopstick feast.

Interaction is also a process. It concerns the relationship between an entity and an environment that brings about differential replication. The entity in question must interact with its environment as a cohesive whole (Hull, 1988, 2001). Many cohesive wholes exist in nature, but only a few of them count as interactors in a selection process. According to Hull $(1988,2001)$ chromosomes, gametes, organisms, and possibly populations and species interact as cohesive wholes with their environment in a way that brings about differential replication, i.e., some structures become more common, some more rare.

Just as genes require very strong connections with organisms in order to bring about differential replication, so too do stable cultural components require very strong connections with human individuals and social organizations. Units of culture (e.g. routines) must be strongly connected with their carriers (e.g. firms) for differential replication to occur. In addition, cultural entities must interact as cohesive wholes with their environment in a way that brings about differential replication. However, this interpretation depends critically on the understanding of terms such as 'cohesive' and 'strong'. At this point it is necessary to add some precision to the definition of an interactor. The term 'cohesive whole' indicates that its components stick together and remain 
united. We interpret this to mean that all the components depend critically on the survival of the whole, and that to some degree the components depend on the survival of each other.

If an entity is an interactor, then it must satisfy all of the following minimal conditions (Hodgson and Knudsen, 2004). For each interactor there is a corresponding non-empty 'component' set of replicators, such that the following conditions apply: (1) shared dependence of component replicators: if the interactor perishes, then all its component replicators are also likely to perish, (2) shared organisation of components: every component replicator is hosted by its interactor and principally interacts with the outside world through the interactor, (3) relative independence of noncomponent replicators: the interactor and any non-component replicator are independent in the sense that the survival of one does not largely depend on the survival of the other, and (4) propertydependent replication: the properties of an interactor determines the expected number of successor replicators within a given environment.

Interactors and replicators are particularly important objects of selection, both in nature and at the social level. In nature, genes are paradigm examples of replicators and organisms of interactors. However, according to the hierarchical perspective on selection (Lewontin, 1970), which is widely accepted today, there is selection at multiple interdependent levels, including cells, organisms, groups, populations, species, and ecological communities. Any of these levels of biological organization count as interactors (Brandon, 1998, 1999; Hull, 1988, 2001) because entities at each of these levels interact as cohesive wholes with their environment in a way that brings about differential replication, i.e., some structures become more common, some more rare. Whereas interactors are present at each level of biological organization, only a few entities have evolved to fulfil a specialised function as a replicator. The gene remains the paradigm example. In consequence, interactors at multiple levels may correspond to entities at lower levels that both fulfil the role of being interactors and replicators.

\subsubsection{Social Replicators and Interactors}

Little is known about interactors and replicators at the social level. There is an emerging consensus that habits are fundamental, individual-level replicators whereas routines count as organization level replicators. Habits and routines are persistent containers of encoded instructions for behaviour or thought. When this information is reliably passed on, habits and routines can function as replicators (Hodgson, 2001, 2003; Hodgson and Knudsen, 2004; Knudsen, 2001, 2002, 2005; Metcalfe, 1998; Winter and Szulanski, 2001). A very broad empirical literature supports the view that habits and routines have degrees of persistence and copying fidelity that warrant their status as replicating repositories of knowledge and custom (Hodgson, 2005; Knudsen, 2005).

For example, routines and folkways of families and religious communities often emerge in new families and religious communities (Fischer, 1989). In this case, the interaction of cultural groups can cause differential replication of routines. Also the interaction of firms with a particular environment (e.g. other firms in an industry) is commonly viewed as the cause of differential replication of routines, at least in the minimal sense that stable firm-specific traits underlie differential expansion and contraction of firms (Metcalfe, 1998; Metcalfe and Gibbons, 1995; Nelson and Winter, 1982). Different mechanisms of replication are involved when a central unit (Headquarters) uses a template to produce a large number of copies of an outlet that delivers a service or produces a product (Winter and Szulanski, 2001). In this case, the interaction of Headquarters with its competitors leads to differential replication of the template. Apparently, social organizations can function as interactors that replicate routines in a number of ways. 
Our understanding in these terms about replicators and interactors in the social domain is presently rather incomplete. Little attention has been directed towards the obvious requirement in generative selection of defining corresponding pairs of replicators and interactors. Contributors to memetics (Dawkins, 1976; Blackmore, 1999; Aunger, 2002) and students of cultural evolution (Boyd and Richerson, 1985; Durham, 1991) have primarily focussed on the identification of replicators, while downplaying the need to firmly establish correspondence between replicator and interactor.

Hodgson and Knudsen (2004) made a case for regarding firms and similarly cohesive institutions as interactors that correspond to replicating routines. However, this is only a first step towards a more systematic identification of a hierarchy of interactors and replicators in the social domain. While the identification of interactors and replicators is arguably required for the study of generative selection processes, little is known about the identity of the entities that count as corresponding interactors and replicators in a hierarchy of social organization. Possibly, habits and routines are replicators that give rise to the remarkable persistence of individual and organizational behaviour that have been reported in recent empirical studies (Cohen and Bacdyan, 1994; Wood and Quinn, 2004). However, little is known about the different kinds of selection processes that shape the distribution of replicating habits and routines.

In summary, replication and interaction are critical because they are mechanisms required to produce generative selection. Both human individuals and social organizations are social level interactors. The social interaction of human individuals causes differential replication of units of culture such as habits. Families, religious groups, firms and other social groups are higher-level interactors. The interaction of social organizations causes differential replication of higher-level units of culture such as routines. Possibly, cultural units such as folkways, customs, conventions, and institutions replicate. Also technologies and ideas might replicate. A challenge before us is to identify the role of various units of culture in selection processes and the multiple levels at which social selection processes take place. We should aim to outline the different kinds of selection processes that are present at each level and the way they are related. To begin addressing this issue, the present article offers a general definition of selection and two sharp concepts of selection that are consistent with it. 


\section{References}

Aldrich, Howard E. (1999) Organizations Evolving (London: Sage Publications).

Aldrich, Howard E. (2004) 'Entrepreneurship', in Neil Smelser and Richard Swedberg (eds), Handbook of Economic Sociology (Princeton University Press).

Andersen, Esben Sloth (2004) 'Population Thinking, Price's Equation and the Analysis of Economic Evolution', Evolutionary and Institutional Economics Review, 2004, 1(1), November, pp. 127-48.

Aunger, Robert (2002) The Electric Meme: A New Theory of How We Think (New York: Free Press).

Blackmore, Susan (1999) The Meme Machine (Oxford: Oxford University Press).

Boyd, Robert and Richerson, Peter J. (1985) Culture and the Evolutionary Process (Chicago: University of Chicago Press).

Brandon, Robert N. (1998) 'The Levels of Selection: A Hierarchy of Interactors', in Hull, D. L. and Ruse, M. (eds) (1998) The Philosophy of Biology (Oxford: Oxford University Press), pp. 176197.

Brandon, Robert N. (1999) 'The Units of Selection Revisited: The Modules of Selection', Biology and Philosophy, 14, pp. 167-180.

Cohen, Michael D. and Bacdayan, Paul (1994) 'Organizational Routines are Stored as Procedural Memory - Evidence from a Laboratory Study’, Organization Science, 5(4), November, pp. 55468.

Commons, John R. (1934) Institutional Economics - Its Place in Political Economy (New York: Macmillan).

Darwin, Charles R. (1859) On the Origin of Species by Means of Natural Selection, or the Preservation of Favoured Races in the Struggle for Life (London: Murray).

Dawkins, Richard (1976) The Selfish Gene, (Oxford: Oxford University Press).

Durham, William H. (1991) Coevolution. Genes, Culture and Human Diversity (Stanford, CA: Stanford University Press).

Fischer, David Hackett (1989) Albions Seed. Four Brittan Folkways in America (New York and Oxford: Oxford University Press).

Foster, J. and Metcalfe, J. S. (2001) Frontiers of Evolutionary Economics (Cheltenham: Elgar).

Frank, Steven A. (1997) 'Optimal Parameters for Variation and Selection in Learning and Development', Journal of Theoretical Biology, 194, pp. 31-29.

Frank, Steven A. (1998) Foundations of Social Evolution (Princeton, NJ: Princeton University Press).

Godfrey-Smith, Peter (2000) 'The Replicator in Retrospect', Biology and Philosophy, 15, pp. 40323.

Hannan, Michael T., Freeman John (1989) Organizational Ecology (Cambridge, MA: Harvard University Press). 
Henrich, Joseph (2004) 'Cultural Group Selection, Coevolutionary Processes and Large-Scale Cooperation', Journal of Economic Behavior and Organization, 53(1), February, pp. 3-35.

Hodgson, Geoffrey M. (2001) 'Is Social Evolution Lamarckian or Darwinian?' in Laurent, John and Nightingale, John (eds) (2001) Darwinism and Evolutionary Economics (Cheltenham: Edward Elgar), pp. 87-118.

Hodgson, Geoffrey M. (2002) 'Darwinism in Economics: From Analogy to Ontology', Journal of Evolutionary Economics, 12(2), June, pp. 259-81.

Hodgson, Geoffrey M. (2003) 'The Mystery of the Routine: The Darwinian Destiny of $A n$ Evolutionary Theory of Economic Change', Revue Économique, 54(2), Mars, pp. 355-84.

Hodgson, Geoffrey M., Knudsen, Thorbjørn (2004) 'The Firm as an Interactor: Firms as Vehicles for Habits and Routines', Journal of Evolutionary Economics, 14(3), pp. 281-307.

Hodgson, Geoffrey M. and Knudsen, Thorbjørn (forthcoming) 'Dismantling Lamarckism: Why Descriptions of Socio-Economic Evolution as Lamarckian are Misleading', Journal of Evolutionary Economics (forthcoming).

Hofbauer, Josef, Sigmund, Karl (1998) Evolutionary Games and Population Dynamics (Cambridge: Cambridge University Press).

Hull, David L. (1988) Science as a Process: An Evolutionary Account of the Social and Conceptual Development of Science (Chicago: University of Chicago Press).

Hull, David L. (2001) Science and Selection. Essays on Biological Evolution and the Philosophy of Science (Cambridge: Cambridge University Press).

Hull, David L., Langman, Rodney E., and Glenn, Sigrid S. (2001) 'A General Account of Selection: Biology, Immunology and Behavior', Behavioral and Brain Sciences, 24(3), pp. 511-28.

Katz, Jerome A., Gartner, William B. (1988) 'Properties of emerging organizations', Academy of Management Review, 13, pp. 429-441.

Knudsen, T. (2001) 'Nesting Lamarckism within Darwinian Explanations: Necessity in Economics and Possibility in Biology?', in J. Laurent and J. Nightingale (eds.) Darwinism and Evolutionary Economics, pp. 121-159 (Cheltenham: Edward Elgar).

Knudsen, Thorbjørn (2002) 'Economic Selection Theory', Journal of Evolutionary Economics, 12, pp. 443-470.

Knudsen, Thorbjørn (2004) 'General Selection Theory and Economic Evolution: The Price Equation and the Replicator/Interactor Distinction', Journal of Economic Methodology, 11(2), June, pp. 147-73.

Knudsen, Thorbjørn (2006) 'Organizational Routines in Evolutionary Theory', in Becker, Markus (ed.) The Handbook of Organizational Routines (forthcoming).

Landa, A. Gudvangen, K., Swenson, J.E., Roskaft, E. (1999) 'Factors Associated with Wolverine Gulo gulo Predation on Domestic Sheep', The Journal of Applied Ecology, 36(6), pp. 963-973

Lewontin, Richard (1970) 'The Units of Selection', Annual Review of Ecology and Systematics 1, pp. 1-17.

Maynard Smith, John and Szathmáry, Eors (1999) The Origins of Life: From the Birth of Life to the Origin of Language (Oxford: Oxford University Press). 
McMillan, John (2002) Reinventing the Bazaar. A Natural History of Markets (New York and London: W.W. Norton and Company).

Metcalfe, J. Stanley (1998) Evolutionary Economics and Creative Destruction. The Graz Schumpeter Lectures (London: Routledge).

Metcalfe, J.S. and Gibbons, M. (1995). Technological Variety and the Process of Competition (Reprinted from Economie Appliqué, XXXIX, 1986). In Hodgson, G.M. (ed.): Economics and Biology. Aldershot: Edward Elgar, 428-455.

Nelson, Richard R., Winter, Sidney G. (1982) An Evolutionary Theory of Economic Change (Cambridge, MA: Harvard University Press).

Pepper, John W., Knudsen, Thorbjørn (2001) 'Selection Without Multiple Replicators?', Behavioral and Brain Sciences, 24(3): p. 550.

Price, George R. (1995) ‘The Nature of Selection', Journal of Theoretical Biology, 175(3), pp. 389396.

Sober, Elliott (1984) The Nature of Selection: Evolutionary Theory in Philosophical Focus (Cambridge, MA; MIT Press).

Sperber, Dan (2000) 'An Objection to the Memetic Approach to Culture', in Aunger, Robert (ed.) (2000) Darwinizing Culture: The Status of Memetics as a Science (Oxford and New York: Oxford University Press), pp. 162-73.

Sterelny, Kim, Smith, Kelly C. and Dickison, Michael (1996) 'The Extended Replicator', Biology and Philosophy, 11, pp. 377-403.

Szathmáry, Eörs, Maynard Smith, John (1997) 'From Replicators to Reproducers: the First Major Transitions Leading to Life', Journal of Theoretical Biology, 187(4), pp. 555-571.

Tushman, M.L. and Anderson, P. (1986): Technological Discontinuities and Organizational Environments, Administrative Science Quarterly, 31, 439-465.

Vrba, E.S. and Gould, S.J. (1986) 'The Hierarchical Expansion of Sorting and Selection: Sorting and Selection Cannot be Equated', Paleobiology, 12, pp. 217-228.

Winter, Sidney G. (1971) 'Satisficing, Selection, and The Innovating Remnant', The Quarterly Journal of Economics, 85(2), pp. 237-261.

Winter, Sidney G., Szulanski, Gabriel (2001) 'Replication as Strategy', Organization Science, 12(6), pp. 730-743.

Wood, Wendy, Quinn, Jeffrey M. (2004) The Power of Repetition in Daily Life: Habits and Intentions Guide Action (Durham, N.C.: Unpublished manuscript, Duke University). 


\section{Appendix: Price's (1995) Definition of Selection}

According to Price (1995), selection is the act or process of producing a corresponding set. Given a set $P$ containing $\omega_{i}$ amounts of $I$ distinct elements $p_{i}$, which have the properties $x_{i}$, Price (1995) defines a corresponding set as follows:

We will say that a set $P^{\prime}$ is a corresponding set to a set $P$ if there exists a one-to-one correspondence such that, for each member $p_{i}$ of $P$, there is a corresponding member $p_{i}{ }^{\prime}$ of $P^{\prime}$ which (if not empty) is composed partly or wholly of the same material as $p_{i}$, or has been derived directly from $p_{i}$, or contains one or more replicas of $p_{i}$ or some part of $p_{i}$ or has some other special close relation to $p_{i}$. (Price, 1995: 392).

Using this definition of a corresponding set, Price (1995: 392) defines selection as: 'Selection on a set $P$ in relation to a property $x$ is the act or process of producing a corresponding set $P$ ' in a way such that the amounts $\omega_{i}{ }^{\prime}$ (or some function of them such as the ratios $\omega_{i}{ }^{\prime} / \omega_{i}$ ) are non-randomly related to the corresponding $x_{i}$ values.'

The terminology introduced by Price (1995) yields a useful statistical definition of selection. Let $P$ be a set containing $\omega_{i}$ amounts of $I$ distinct elements which have the properties $x_{i}$. A transformation $P \rightarrow P^{\prime}$ (possibly the identity transformation) results in a second set $P^{\prime}$. The set $P^{\prime}$ contains $\omega_{i}{ }^{\prime}$ amounts of $I$ distinct elements with properties $x_{i}{ }^{\prime}$. The transformation $P \rightarrow P^{\prime}$ is termed a selection process that gives rise to the effect $X \rightarrow X^{\prime}$ in a population property $X$ related to property $x$ of the individual set members. This effect $X \rightarrow X^{\prime}$ can be calculated as the change in the average value (Price, 1995; Frank, 1997):

$$
\Delta X=X^{\prime}-X=\sum \omega_{i}^{\prime} x_{i}{ }^{\prime}-\sum \omega_{i} x_{i} \Rightarrow e \Delta X=\operatorname{Cov}\left(e_{i}, x_{i}\right)+E\left(e_{i} \Delta x_{i}\right), \quad \omega_{i}{ }^{\prime}=\omega_{i} e_{i} / e,
$$

where $e_{i}$ is the fitness of element $i$ in the set $P$ and $e$ is average fitness of the set $P$. Selection is present whenever $\operatorname{Cov}\left(e_{i}, x_{i}\right)$ differs significantly from zero. By contrast, a transmission effect is present whenever $E\left(e_{i} \Delta x_{i}\right)$ differs significantly from zero. In social populations, a transmission effect can be referred to as an individual-level exploration or innovation effect. A selection or a transmission effect refers to a change in the first moment of the trait distribution, i.e., selection is a change in the average trait according to the population in question. The Price Equation thus provides a useful solution to the definition and empirical verification of possible selection effects in a population of interest. As the reader can verify, it is straightforward to recursively expand the above expression to include multiple hierarchical layers of selection (use the expectation term for expansion). A further (not too challenging) issue concerns the expansion of the Price Equation to encompass selection on higher moments than the first. 